\title{
Right Ventricle Dilation Detected on Point-of-Care Ultrasound Is a Predictor of Poor Outcomes in Critically III Patients With COVID-19
}

\author{
Pooja Belligund, MD; David Lee, MD; Mehrala Balasubramaniam, MD; Suchit Khanijao, MD; \\ Dushyant Damania, MD; Nishant Vallumsetla, MD; Qasim Sajawal, MD; Brais Perez-Gandara, MD; \\ Jessica Perez-Perez, MD; Isaac Shalom, MD; Gangacharan Dubey, MD; Sarah Sanghavi, MD; \\ Chen Lu, MD; Cristina Mitre, MD; Joe Zein, MD, PhD; and Mohammad Al-Ajam, MD
}

Background: During the COVID-19 pandemic, the need for judicious use of diagnostic tests and to limit personnel exposure has led to increased use and dependence on point-ofcare ultrasound (POCUS) examinations. We reviewed POCUS findings in patients admitted to the intensive care unit (ICU) for acute respiratory failure with COVID-19 and correlated the findings to severity of illness and 30-day outcomes.

Methods: Patients admitted to the ICU in March and April 2020 were reviewed for inclusion (acute hypoxemic respiratory failure secondary to COVID-19 pneumonia; documentation of POCUS findings).

Results: Forty-three patients met inclusion criteria. B lines and pleural thickening were associated with a lower $\mathrm{PaO}_{2} /$ $\mathrm{FiO}_{2}$ by $71\left(P=.005\right.$; adjusted $\left.R^{2}=0.24\right)$. Right ventricle (RV) dilation was more common in patients with 30-day mortality $(P=.02)$ and was a predictor of mortality when adjusted for hypertension, diabetes mellitus, and age (odds ratio, 12.0; $P=.048$ ). All patients with RV dilation had bilateral $B$ lines with pleural irregularities.

Conclusions: Although lung ultrasound abnormalities are prevalent in patients with severe disease, RV involvement seems to be predictive of outcomes. Further studies are needed to discern the etiology and pathophysiology of RV dilation in COVID-19.
Author affiliations can be found at the end of the article.

Correspondence:

Pooja Belligund

(pooja.belligund@va.gov)

Fed Pract. 2021;38(9) Published online September 12. doi:10.12788/fp.0177
$\mathrm{P}$ oint-of-care ultrasound (POCUS) is increasingly being used by critical care physicians to augment the physical examination and guide clinical decision making, and several protocols have been established to standardize the POCUS evaluation. ${ }^{1}$ During the COVID19 pandemic, POCUS has been a valuable tool as standard imaging techniques were used judiciously to minimize exposure of personnel and use of personal protective equipment (PPE). ${ }^{2}$

In the US Department of Veterans Affairs (VA) New York Harbor Healthcare System (VANYHHS) intensive care unit (ICU) on initial clinical examination included POCUS, which was helpful to examine deep vein thromboses, cardiac function, and the presence and extent of pneumonia. An international expert consensus on the use of POCUS for COVID-19 published in December 2020 called for further studies defining the role of lung and cardiac ultrasound in risk stratification, outcomes, and clinical management. ${ }^{3}$

The objective of this study was to review POCUS findings and correlate them with severity of illness and 30-day outcomes in critically ill patients with COVID-19.

\section{METHODS}

The study was submitted to and reviewed by the VANYHHS Research and Development committee and study approval and informed consent waiver was granted. The study was a retrospective chart review of patients admitted to the VANYHHS ICU between March and April 2020, a tertiary health care center designated as a COVID19 hospital.

Patients admitted to the ICU aged $>18$ years with a diagnosis of acute hypoxemic respiratory failure, diagnosis of COVID19 , and documentation of POCUS findings in the chart were included in the study. A patient was considered to have a COVID-19 diagnosis following a positive SARS-CoV-2 polymerase chain reaction test documented in the electronic health record (EHR). Acute respiratory failure was defined as hypoxemia $<94 \%$ and the need for either supplemental oxygen by nasal cannula $>2 \mathrm{~L}$ min, high flow nasal cannula, noninvasive ventilation, or mechanical ventilation.

To minimize personnel exposure, initial patient evaluations and POCUS examinations were performed by the most senior personnel (ie, fellowship trained, board-certified pulmonary critical care attending physicians or pulmonary and critical care fellowship 
TABLE 1 Baseline Characteristics

\begin{tabular}{|c|c|c|c|}
\hline Characteristics & Discharged Within $30 \mathrm{~d}$ & Mortality Within $30 \mathrm{~d}$ & $P$ value \\
\hline No. & 14 & 24 & \\
\hline Age, mean (SD), y & $64(11)$ & $71(9)$ & .03 \\
\hline Female, No. (\%) & $1(7)$ & $1(4)$ & .99 \\
\hline Hypertension, No. (\%) & $11(77)$ & $20(83)$ & .99 \\
\hline Chronic obstructive pulmonary disease, No. (\%) & $0(0)$ & $6(25)$ & .12 \\
\hline Diabetes mellitus, No. (\%) & $5(36)$ & $16(67)$ & .13 \\
\hline Smoking history, No. (\%) & $6(43)$ & $11(46)$ & .10 \\
\hline White blood cells, mean (SD), K/uL & $14.5(6)$ & $31(10)$ & .02 \\
\hline C-reactive protein, mean (SD), $\mathrm{mg} / \mathrm{dL}$ & $10(7)$ & $23(10)$ & $<.001$ \\
\hline Lactate, mean (SD), mmol/L & $1.96(0.35)$ & $2.76(1.97)$ & .21 \\
\hline Interleuken-6, mean (SD), pg/mL & $265(225)$ & $354(294)$ & .46 \\
\hline Ferritin, mean (SD), $\mathrm{ng} / \mathrm{mL}$ & $1062(574)$ & $1302(508)$ & .19 \\
\hline Troponin, mean (SD), ng/mL & $0.12(0.11)$ & $3.17(11)$ & .31 \\
\hline D-dimer mean (SD), $\mathrm{ng} / \mathrm{mL}$ & $7576(13,173)$ & $11,464(15,342)$ & .44 \\
\hline $\mathrm{PaO}_{2} / \mathrm{FiO}_{2}$, mean (SD) & $135.6(64)$ & $88(41)$ & .02 \\
\hline B lines with irregular pleura (B'), No. (\%) & $7(50)$ & $18(75)$ & .16 \\
\hline Reduced left ventricular function, No. (\%) & $3(21)$ & $3(12.5)$ & .79 \\
\hline B lines without irregular pleura (B), No. (\%) & $12(86)$ & $20(83)$ & .99 \\
\hline Right ventricular dilation, No. (\%) & $0(0)$ & $10(42)$ & .02 \\
\hline Mechanical ventilation, No. (\%) & $6(43)$ & $23(96)$ & .001 \\
\hline
\end{tabular}

trainees). Three members of the team had certification in advanced critical care echocardiography by the National Board of Echocardiography and oversaw POCUS imaging. POCUS examinations were performed with a GE Heathcare Venue POCUS or handheld unit. After use, ultrasound probes and ultrasound units were disinfected with wipes designated by the manufacturer and US Environmental Protection Agency for use during the COVID-19 pandemic.

The POCUS protocol used by members of the team was as follows: POCUS lungat least 2 anterior fields and 1 posterior/lateral field looking at the costophrenic angle on each hemithorax with a phased array or curvilinear probe. A linear probe was used to look for subpleural changes per physician discretion. ${ }^{4,5}$ Lung ultrasound findings in anterior lung fields were documented as A lines, B lines (as defined by the bedside lung ultrasound in emergency [BLUE] protocol) anterior pleural abnormalities or consolidations. ${ }^{4,5}$ The costophrenic point findings were documented as presence of consolidation or pleural effusion.

The POCUS cardiac examination consisted of parasternal long and short axis views, apical 4 chamber view, subcostal and inferior vena cava (IVC) view. Left ventricular (LV) ejection fraction was visually estimated as reduced or normal. Right ventricular (RV) dilation was considered present if RV size approached or exceeded LV size in the apical 4 chamber view. RV dysfunction was considered present if in addition there was flattening of interventricular septum, RV free wall hypokinesis or reduced tricuspid annular plane systolic excursion (TAPSE). ${ }^{6}$ IVC was documented 
TABLE 2 POCUS Findings by Mechanical Ventilation Need

\begin{tabular}{lccc} 
Characteristics & $\begin{array}{c}\text { Noninvasive } \mathbf{O}^{2} \\
\text { Therapy, No. (\%) }\end{array}$ & $\begin{array}{c}\text { Mechanical } \\
\text { Ventilation, No. (\%) }\end{array}$ & $\begin{array}{c}\boldsymbol{P} \\
\text { value }\end{array}$ \\
\hline No. & 9 & 20 & \\
\hline A lines bilaterally/A profile & $1(11)$ & $3(10)$ & .99 \\
\hline B lines bilaterally/B profile & $3(33)$ & $7(24)$ & .91 \\
\hline B lines + pleural irregularities/B' & $5(56)$ & $19(66)$ & .88 \\
\hline Consolidation & $0(0)$ & $1(3)$ & .99 \\
\hline $\begin{array}{l}\text { Left ventricular ejection fraction } \\
\text { reduced }\end{array}$ & $2(22)$ & $4(14)$ & .93 \\
\hline Right ventricular dilation & $0(0)$ & $10(35)$ & .11 \\
\hline 30-day mortality & $1(11)$ & $23(80)$ & .001
\end{tabular}

as collapsible or plethoric by size and respirophasic variability ( $2 \mathrm{~cm}$ and $50 \%$ ). Other POCUS examinations including venous compression were done at the discretion of the treating physician. ${ }^{7}$ POCUS was also used for the placement of central and arterial lines and to guide fluid management. ${ }^{8}$

The VA EHR and Venue image local archives were reviewed for patient demographics, laboratory findings, imaging studies and outcomes. All ICU attending physician and fellow notes were reviewed for POCUS lung, cardiac and vascular findings. The chart was also reviewed for management changes as a result of POCUS findings. Patients who had at minimum a POCUS lung or cardiac examination documented in the EHR were included in the study. For patients with serial POCUS the most severe findings were included.

Patients were divided into 2 groups based on 30-day outcome: discharge home vs mortality for comparison. POCUS findings were also compared by need for mechanical ventilation. Patients still hospitalized or transferred to other facilities were excluded from the analysis. A Student $t$ test was used for comparison between the groups for continuous normally distributed variables. Linear and stepwise regression models were used to evaluate univariate and multivariate associations of baseline characteristics, biomarker, and ultrasound findings with patient outcomes. Analyses were performed using R 4.0.2 statistical software.

\section{RESULTS}

Eighty-two patients were admitted to the VANYHHS ICU in March and April 2020, including 12 nonveterans. Sixty-four had COVID-19 and acute respiratory failure. POCUS findings were documented in $43(67 \%)$ patients. Thirty-nine patients had documented lung examinations, and 25 patients had documented cardiac examinations. Patients were divided into 2 groups by 30 -day outcome (discharge home vs mortality) for statistical analysis. Five patients who were either still hospitalized or had been transferred to another facility were excluded.

Baseline characteristics of patients included in the study stratified by 30-day outcomes are shown in Table 1 . The study group was predominantly male (95\%). Patients with poor 30-day outcomes were older, had higher white blood cell counts, more severe hypoxemia, higher rates of mechanical ventilation and RV dilation (Figures 1, 2, 3, 4, and 5). RV dilation was an independent predictor of mortality (odds ratio [OR], 12.0; $P=.048$ ).

Serial POCUS documented development or progression of RV dilation and dysfunction from the time of ICU admission in 4 of the patients. The presence of $B$ lines with irregular pleura was predictive of a lower arterial pressure of oxygen to fraction of inspired oxygen ratio $\left(\mathrm{PaO}_{2} / \mathrm{FiO}_{2}\right)$ by a value of 71 compared with those without $\mathrm{B}$ lines with irregular pleura $\left(P=.005\right.$, adjusted $R^{2}=$ 0.238 ). All patients with RV dilation had bilateral B lines with pleural irregularities on lung ultrasound. Vascular POCUS detected 4 deep vein thromboses (DVT). ${ }^{7}$ An arterial thrombus was also detected on focused examination. There was a higher mortality in patients who required mechanical ventilation; however, there was no difference in POCUS characteristics between the groups (Table 2).

Two severely hypoxemic patients received systemic tissue plasminogen activator (TPA) after findings of massive RV dilation with signs of volume and pressure overload and clinical suspicion of pulmonary embolism (PE). One of these patients also had a popliteal DVT. Both patients were too unstable to transport for additional imaging or therapies. Therapeutic anticoagulation was initiated on 4 patients with positive DVT examinations. 
In a fifth case an arterial thrombectomy and anticoagulation was required after diminished pulses led to the finding of an occlusive brachial artery thrombus on vascular POCUS.

\section{DISCUSSION}

POCUS identified both lung and cardiac features that were associated with worse outcomes. While lung ultrasound abnormalities were very prevalent and associated with worse $\mathrm{PaO}_{2}$ to $\mathrm{FiO}_{2}$ ratios, the presence of RV dilation was associated most clearly with mortality and poor 30-day outcomes in the critical care setting.

Lung ultrasound abnormalities were pervasive in patients with acute respiratory failure and COVID-19. On linear regression we found that presence with bilateral $\mathrm{B}$ lines and pleural thickening was predictive of a lower $\mathrm{PaO}_{2} / \mathrm{FiO}_{2}$ (coefficient, -70; $P=.005$ ). Our study found that B lines with pleural irregularities, otherwise known as a B' profile per the BLUE protocol, was seen in patients with severe COVID-19. Thus severe acute respiratory failure secondary to COVID-19 has similar lung ultrasound findings as non-COVID-19 acute respiratory distress syndrome (ARDS). 4,5 Based on prior lung ultrasound studies in ARDS, lung ultrasound findings can be used as an alternate to chest radiography for the diagnosis of ARDS in COVID-19 and predict the severity of ARDS. ${ }^{9}$ This has particular implications in overwhelmed and resource poor health care settings.

We found no difference in 30-day mortality based on lung ultrasound findings or profile, probably because of small sample size or because the findings were tabulated as profiles and not differentiated further with lung ultrasound scores. ${ }^{10,11}$ However, there was a significant difference in RV dilation between the 2 groups by 30 days and its presence was found to be a predictor of mortality even when controlled for hypertension and diabetes mellitus $(P=.048)$ with an OR of 12. RV dysfunction in patients with ARDS on mechanical ventilation ranges from 22 to $25 \%$ and is typically associated with high driving pressures. ${ }^{12-14}$ The mechanism is thought to be multifactorial including hypoxemic vasoconstriction in the pulmonary vasculature in addition to the increased trans-
FIGURE 1 Lung Ultrasound, Phased Array Probe With Tissue Harmonics On

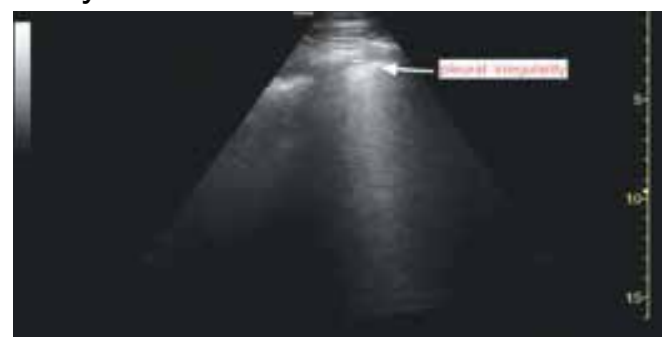

Anterior right lung showing pleural irregularity and thickening.

FIGURE 2 Lung Ultrasound, Phased Array Probe, Anterior Lung Field, Tissue Harmonics Off

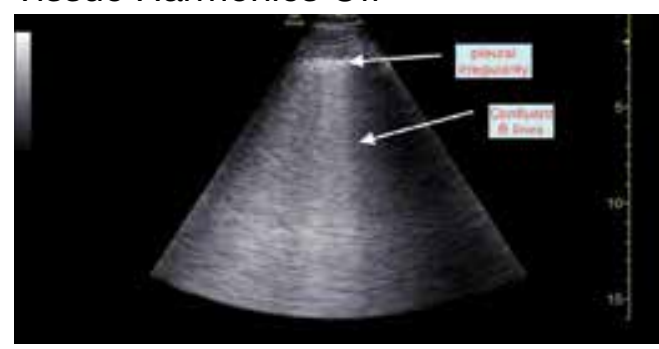

Anterior lung field showing confluent $B$ lines and pleural irregularity.

\section{FIGURE 3 Lung Ultrasound, Linear Probe}

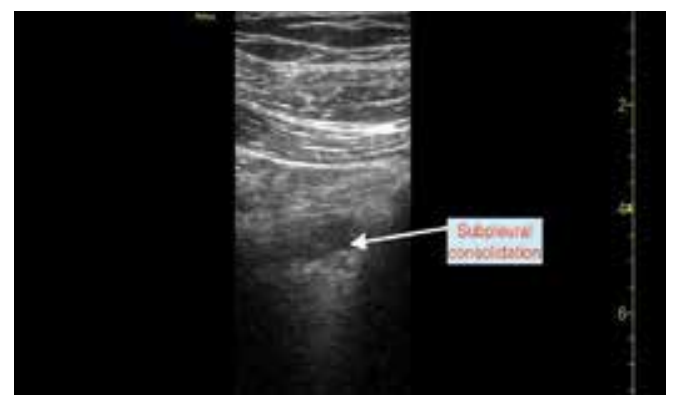

Anterior lung field showing subpleural consolidation.

pulmonary pressure. ${ }^{15}$ While all of the above are at play in COVID-19 infection, there is reported damage to the pulmonary vascular endothelium and resultant hypercoagulability and thrombosis that further increases the RV afterload. ${ }^{16}$

While RV strain and dysfunction indices done by an echocardiographer would be ideal, given the surge in infections and hospitalizations and strain on health care resources, POCUS by the treating or examining clinician was considered the only feasible way to screen a large number of patients. ${ }^{17}$ Identification of RV dilation could influence clinical management in- 
FIGURE 4 Phased Array Probe, Lateral Lung Field, Irregular Pleura With B Lines

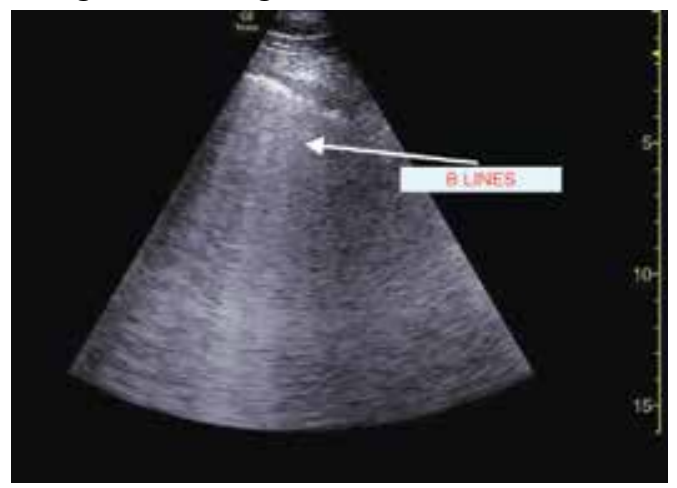

FIGURE 5 Right Ventricular Dilation With Volume and Pressure Overload

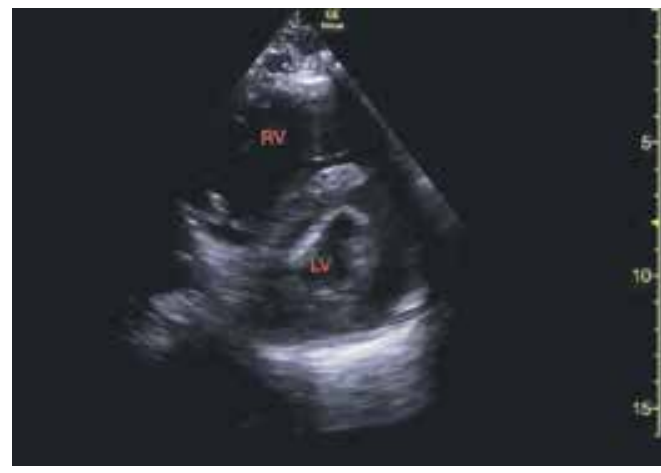

cluding workup for venous thromboembolic disease and optimization of lung protective strategies. Further studies are needed to understand the particular etiology and pathophysiology of COVID-19 associated RV dilation. Given increased thrombosis events in COVID-19 infection we believe a POCUS vascular examination should be included as part of evaluation especially in the presence of increased D-dimers and has been discussed above for its important role in working up RV dilation. ${ }^{18}$

\section{Limitations}

Our study has several limitations. It was retrospective in nature and involved a small group of individuals. There was some variation in POCUS examinations done at the discretion of the examining physician. We did not have a blinded observer independently review all images. Since RV dilation was documented only when RV size approached or exceeded LV size in the apical 4 chamber view repre- senting moderate or severe dilation, we may be underreporting the prevalence in critically ill patients.

\section{CONCLUSIONS}

POCUS is an invaluable adjunct to clinical evaluation and procedures in patients with severe COVID-19 with the ability to identity patients at risk for worse outcomes. B lines with pleural thickening is a sign of severe ARDS and RV dilatation is predictive of mortality. POCUS should be made available to the treating physician for monitoring and risk stratification and can be incorporated into management algorithms.

Visit mdedge.com/fedprac or doi:10.12788/fp.0177 for additional point-of-care ultrasound videos.

\section{Acknowledgments}

We thank frontline healthcare workers and intensive care unit staff of the US Department of Veterans Affairs New York Harbor Healthcare System (NYHHS) for their dedication to the care of veterans and civilians during the COVID-19 pandemic in New York City. The authors acknowledge the NYHHS research and development committee and administration for their support.

\section{Author affiliations}

Pooja Belligund, Isaac Shalom, and David Lee are all staff physicians in the Division of Pulmonary and Critical Medicine; Gangacharan Dubey is the Director of the Intensive Care Unit in the Division of Pulmonary and Critical Care Medicine, and Mohammad Al-Ajam is Chief of the Division of Pulmonary and Critical Care Medicine. Cristina Mitre is a Staff Physician in the Division of Cardiology; all at US Department of Veterans Affairs New York Harbor Healthcare System in Brooklyn, New York. All are Assistant Professors of Medicine at SUNY Downstate Health Sciences University in Brooklyn. Mehrala Balasubramaniam, Suchit Khanijao, Dushyant Damania, Nishant Vallumsetla, and Qasim Sajawal are current or former Fellows in the Division of Pulmonary and Critical Care Medicine; Brais Perez-Gandara and Jessica Perez-Perez are Residents in the Department of Internal Medicine; Chen Lu is a Fellow in the Division of Cardiology; all at SUNY Downstate Health Sciences University in Brooklyn. Sarah Sanghavi is a Nephrology and Critical Care Staff Physician at the VA Puget Sound Health Care System in Seattle, Washington. Joe Zein is a Staff Physician and Associate Professor Medicine at the Cleveland Clinic, Respiratory Institute in Beachwood, Ohio.

\section{Authors roles}

Corresponding author, conceptualization, writing original draft, data collection and analysis: Pooja Belligund; conceptualization, methodology, formal analysis: Mohammad Al-Ajam, Cristina Mitre, Joe Zein, Dushyant Damania, and David Lee; data collection and analysis: Brais Perez Gandara and Jessica Perez-Perez; review and editing: Nishant Vallumsetla, Mehrala Balasubramaniam, Suchit Khanijao, Qasim Sajawal, Sarah Sanghavi, Isaac Shalom, Gangacharan Dubey, and Chen Lu.

\section{Author disclosures}

The authors report no actual or potential conflicts of interest with regard to this article. 


\section{Disclaimer}

The opinions expressed herein are those of the authors and do not necessarily reflect those of Federal Practitioner, Frontline Medical Communications Inc., the US Government, or any of its agencies.

\section{References}

1. Cardenas-Garcia J, Mayo PH. Bedside ultrasonography for the intensivist. Crit Care Clin. 2015;31(1):43-66. doi:10.1016/j.ccc.2014.08.003

2. Vetrugno L, Baciarello M, Bignami E, et al. The "pandemic" increase in lung ultrasound use in response to Covid-19: can we complement computed tomography findings? A narrative review. Ultrasound J. 2020;12(1):39. Published 2020 Aug 17. doi:10.1186/s13089-020-00185-4

3. Hussain A, Via G, Melniker L, et al. Multi-organ pointof-care ultrasound for COVID-19 (PoCUS4COVID): international expert consensus. Crit Care. 2020;24(1):702. Published 2020 Dec 24. doi:10.1186/s13054-020-03369-5

4. Lichtenstein DA, Mezière GA. Relevance of lung ultrasound in the diagnosis of acute respiratory failure: the BLUE protocol [published correction appears in Chest. 2013 Aug;144(2):721]. Chest. 2008;134(1):117-125. doi:10.1378/chest.07-2800

5. Volpicelli G, Elbarbary M, Blaivas M, et al. International evidence-based recommendations for point-of-care lung ultrasound. Intensive Care Med. 2012;38(4):577-591. doi:10.1007/s00134-012-2513-4

6. Narasimhan M, Koenig SJ, Mayo PH. Advanced echocardiography for the critical care physician: part 1. Chest. 2014;145(1):129-134. doi:10.1378/chest.12-2441

7. Kory PD, Pellecchia CM, Shiloh AL, Mayo PH, DiBello C, Koenig S. Accuracy of ultrasonography performed by critical care physicians for the diagnosis of DVT. Chest. 2011;139(3):538-542. doi:10.1378/chest.10-1479

8. Bentzer P, Griesdale DE, Boyd J, MacLean K, Sirounis $\mathrm{D}$, Ayas NT. Will this hemodynamically unstable patient respond to a bolus of intravenous fluids? JAMA. 2016;316(12):1298-1309. doi:10.1001/jama.2016.12310

9. See KC, Ong V, Tan YL, Sahagun J, Taculod J. Chest radiography versus lung ultrasound for identification of acute respiratory distress syndrome: a retrospective observa- tional study. Crit Care. 2018;22(1):203. Published 2018 Aug 18. doi:10.1186/s13054-018-2105-y

10. Deng $Q$, Zhang $Y$, Wang $H$, et al. Semiquantitative lung ultrasound scores in the evaluation and follow-up of critically ill patients with COVID-19: a single-center study. Acad Radiol. 2020;27(10):1363-1372. doi:10.1016/j.acra.2020.07.002

11. Brahier T, Meuwly JY, Pantet $\mathrm{O}$, et al. Lung ultrasonography for risk stratification in patients with COVID-19: a prospective observational cohort study [published online ahead of print, 2020 Sep 17]. Clin Infect Dis. 2020;ciaa1408. doi:10.1093/cid/ciaa1408

12. Vieillard-Baron A, Schmitt JM, Augarde R, et al. Acute cor pulmonale in acute respiratory distress syndrome submitted to protective ventilation: incidence, clinical implications, and prognosis [published correction appears in Crit Care Med. 2002 Mar;30(3):726]. Crit Care Med. 2001;29(8):1551-1555. doi:10.1097/00003246-200108000-00009

13. Boissier F, Katsahian S, Razazi K, et al. Prevalence and prognosis of cor pulmonale during protective ventilation for acute respiratory distress syndrome. Intensive Care Med. 2013;39(10):1725-1733. doi:10.1007/s00134-013-2941-9

14. Jardin F, Vieillard-Baron $A$. Is there a safe plateau pressure in ARDS? The right heart only knows. Intensive Care Med. 2007;33(3):444-447. doi:10.1007/s00134-007-0552-z

15. Repessé X, Vieillard-Baron A. Right heart function during acute respiratory distress syndrome. Ann Transl Med 2017;5(14):295. doi:10.21037/atm.2017.06.66

16. Abou-Ismail MY, Diamond A, Kapoor S, Arafah Y, Nayak L. The hypercoagulable state in COVID-19: Incidence, pathophysiology, and management [published correction appears in Thromb Res. 2020 Nov 26]. Thromb Res. 2020;194:101-115. doi:10.1016/j.thromres.2020.06.029

17. Kim J, Volodarskiy A, Sultana R, et al. Prognostic utility of right ventricular remodeling over conventional risk stratification in patients with COVID-19. J Am Coll Cardiol. 2020;76(17):1965-1977. doi:10.1016/j.jacc.2020.08.066

18. Al-Samkari H, Karp Leaf RS, Dzik WH, et al. COVID-19 and coagulation: bleeding and thrombotic manifestations of SARS-CoV-2 infection. Blood. 2020;136(4):489-500. doi:10.1182/blood.2020006520 